\title{
NEUTRONIC MODELING OF A FAST CORE WITH MODERATING MATERIALS USING APOLLO3 ${ }^{\circledR}$ CODE
}

\author{
M. Zajaczkowski ${ }^{1}$, J.-M. Palau ${ }^{1}$, V. Pascal ${ }^{1}$, C. de Saint Jean ${ }^{2}$ \\ ${ }^{1}$ CEA/Cadarache DEN/DER, 13108 Saint-Paul-lez-Durance, France \\ ${ }^{2}$ CEA/DAM DIF/DPTA/SPN, Bruyeres-le-Chatel 91297 Arpajon, France \\ maciej.zajaczkowski@cea.fr
}

\begin{abstract}
In order to improve passive safety of Sodium-cooled Fast Reactors The French Alternative Energies and Atomic Energy Commission (CEA) has proposed a new core design called CADOR - an SFR core with enhanced Doppler reactivity feedback. One of its most important design features is the introduction of solid moderating materials inside each fuel assemblies to slightly decrease the average neutron energy. The article focuses on development and validation of a neutronics calculation scheme able to produce accurate results in case of CADOR and other fast cores with moderating materials. The study uses two different fuel assembly models moderated by metallic beryllium and zirconium hydride $\left(\mathrm{ZrH}_{2}\right)$ respectively The study includes discussion of neutron scattering treatment and different ways of spatial homogenization and energy condensations. The results indicate that the accurate scattering treatment leads to much better estimation of Doppler constant, especially in case of $\mathrm{ZrH}_{2}$ moderated core. By using combined deterministicMonte Carlo calculation scheme we are able to quantify the biases on global reactivity, reactivity feedbacks and control rod worth. We demonstrate that spatial homogenization plays a more important role in case of moderated CADOR assemblies and thus preserving certain level of heterogeneity within fuel assemblies can lower the calculation bias significantly.
\end{abstract}

KEYWORDS: SFR, CADOR, APOLLO3 ${ }^{\circledR}$, moderation, resonance upscattering

\section{INTRODUCTION}

The Sodium-cooled Fast Reactor (SFR) is arguably one of the most developed and researched Generation 4 reactor concepts. The design provides advantages such as improved fuel economy and lower nuclear waste production. On the other hand addressing safety issues such as positive void coefficient, low Doppler Effect feedback and narrow margin to prompt criticality is a challenging task. In search of safer SFR core design, CEA has launched a "CADOR" research project . The aim is to design a core with more robust passive safety features by increasing the magnitude of Doppler reactivity feedback [1]. One of the project's key changes compared to a standard SFR is addition of small amounts of neutron moderator inside the core. While the resulting neutron spectrum is still close to the one of a typical SFR, the neutron population in the resonance energy range is visibly increased (Figure 1). However, due to strong local variations of neutron energy, higher 


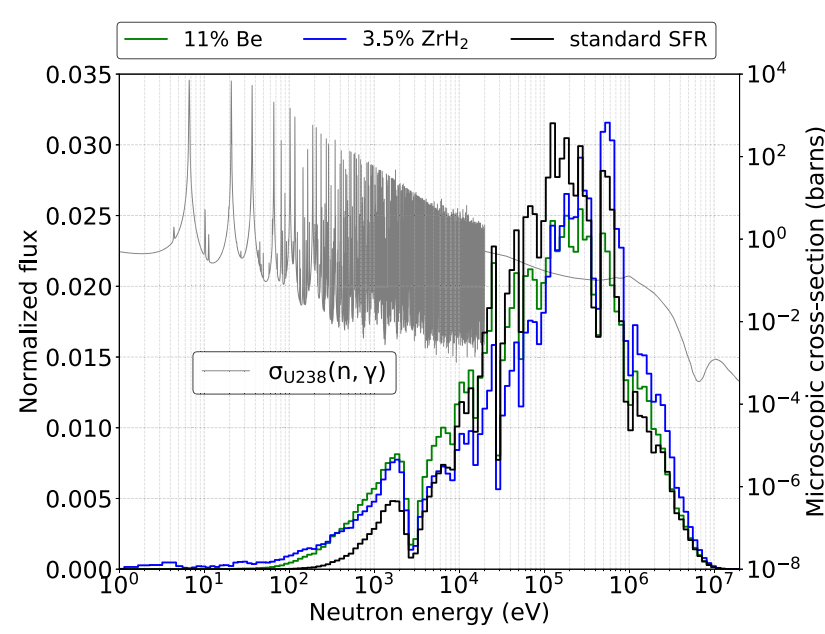

Figure 1: Neutron spectrum comparison for standard SFR and two CADOR assemblies

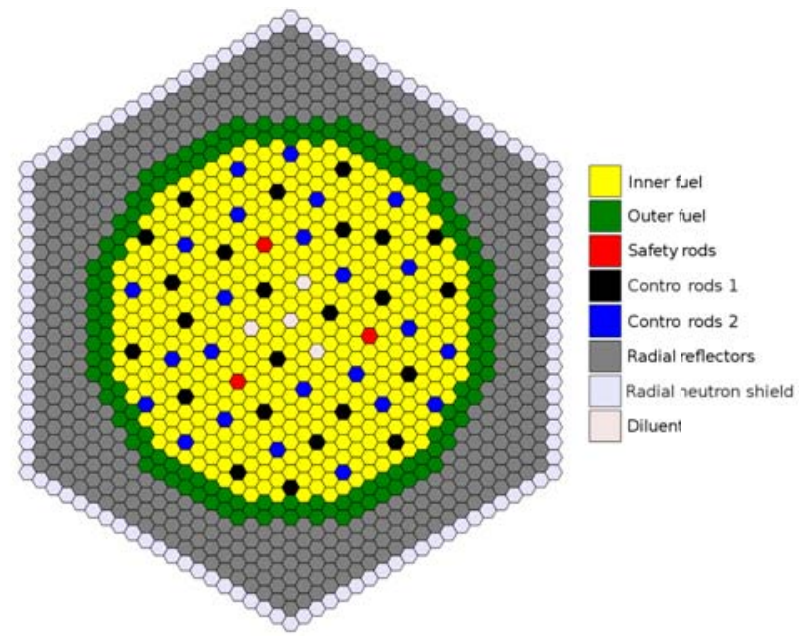

Figure 2: Core layout

importance of resonances absorptions and scatterings utilization of standard calculation methods used for SFR may not be accurate enough. In this study we focus on development and validation of a deterministic neutronics calculation scheme for APOLLO3 ${ }^{\circledR}$ code suitable for calculations of CADOR and other cores where additional moderation is used alongside with fast spectrum. We want to ensure that the numerical models we use can adequately account for phenomena specific to this kind of cores.

\section{CADOR CORE GEOMETRIES}

For this paper we chose a medium size $1500 \mathrm{MW}_{\text {th }}$ CADOR core geometry (Figure 2). The calculations were done for three different version of the core: 1) CADOR with $10.5 \%$ volume content of metallic beryllium per assembly, 2) CADOR with 3.5\% volume content of zirconium hydride $\left(\mathrm{ZrH}_{2}\right)$ per assembly and 3) core without neutron moderator, representing standard SFR approach. The moderator present in first two cases is used in a form of cylindrical rods inserted into cladding in place of some of the fuel pins. This way the shape of fuel assembly remains unchanged and the risk of interaction between moderator and other components is limited. The use of two different CADOR assemblies allows us to draw more general conclusions about the impacts of moderation, as the two moderators exhibit very different properties. Moderation provided by beryllium is less efficient, due to relatively high atomic mass, however it can be distributed more evenly in the assembly. On the other hand $\mathrm{ZrH}_{2}$ is a very strong moderator, thanks to high concentration of hydrogen atoms, but as a result it has to be distributed unevenly in small amounts. The amount of moderators was chosen so that the Doppler feedback in both cases was almost equal.

The whole core can be divided into two sections - the inner core $(\mathrm{C} 1)$ and the outer core $(\mathrm{C} 2)$ with increased $\mathrm{Pu}$ enrichment. Radially the core is surrounded with $\mathrm{MgO}$ reflector, axially the fuel is covered from top and bottom with $20 \mathrm{~cm}$ of fertile blanket and gas plenums. The fissile zone, fertile zone and gas plenums were modeled with exact heterogeneous geometry description; the remaining elements such as rod cluster control assemblies and reflectors were represented as homogeneous materials. 


\section{CALCULATION METHODS}

\subsection{Calculation scheme in $\operatorname{APOLLO3}^{\circledR}$}

APOLLO3 ${ }^{\circledR}[2]$ (abbreviated as A3) is a deterministic neutron transport code developed by CEA. The code is a direct successor of three previous generation codes: APOLLO2, CRONOS2 and ECCO/ERANOS. With a few different solvers at disposal the code is capable of treating structured and unstructured geometries of large and small scales for both fast and thermal reactor applications. The standard calculation scheme of A3 is divided into two steps: the lattice calculation and the core calculation. At the lattice step A3 performs a series of detailed calculations on a set of relatively small geometries, each representing a part of the whole core. At this stage we are able to use exact geometry description without approximations. The aim is, after performing self-shielding and flux calculation, to compute new set of cross-sections, spatially homogenized into fewer regions and condensed into courser energy mesh. The process is performed by integration according to flux-volumes weighting. The lattice calculations in this paper were handled by transport solver TDT [3] with self-shielding performed with $\mathrm{P}_{\mathrm{ij}}$ method and flux calculation performed with MOC. The $\mathrm{C} 1$ assemblies were calculated with $\mathrm{B} 1$ homogeneous leakage model. For the elements such as control rod cluster assemblies or fertile blankets the geometry was organized in a form of clusters with assemblies of interest surrounded by $\mathrm{C} 1$ fuel assemblies. In case of $\mathrm{C} 2$ assemblies and radial reflector a large rectangular cluster of 7 aligned assemblies [4] was used to better simulate the outer core/reflector interaction. All the lattice calculations were performed using fine 1760 group energetic mesh.

Thanks to the cross-section library created in the previous step A3 is able to perform calculation with a full-scale homogenized core model and a broad 33 group energy mesh. The calculation is handled by neutron transport discrete ordinates $\left(\mathrm{S}_{\mathrm{N}}\right)$ solver MINARET [5]. The geometry is discretized into set of axial planes of triangular finite elements (Discontinuous Galerkin FEM). Due to all the simplifications applied through entire calculation scheme the final result of core calculation is affected by a numerical bias.

\subsection{Reference calculation - TRIPOLI-4 ${ }^{\circledR}$ code}

TRIPOLI-4 ${ }^{\circledR}$ [6] (abbreviated as T4) is a Monte Carlo neutron transport code capable of one-step full core calculation with continuous pointwise cross-section and exact geometry representation (later referred to as T4 reference calculation). Because TRIPOLI-4 is free of any approximations with respect to resolution of neutron transport equation it is used as a validation tool for all the deterministic calculations presented in this paper. All the T4 simulations in this study used numbers of neutron histories high enough to ensure a standard deviation of $k_{\text {eff }}$ lower than $5 \mathrm{pcm}$.

\section{IMPACT OF RESONANCE UPSCATTERING}

In this section we discuss the impact of resonance upscattering phenomenon [7] on accuracy of the calculation scheme. For both deterministic and Monte Carlo calculations the accurate treatment of resonance upscattering in fast reactors is often omitted due to its negligible impact. In case of CADOR however the lower average neutron energy suggests that this approach may not be sufficiently accurate. To demonstrate that we compared two different scattering models by performing 
pointwise TRIPOLI-4 calculations. First model, Sampling of the Velocity of the Target (SVT), is a scattering model typically used in fast reactor applications. It assumes low variation of scattering cross-sections with respect to targets (nucleus) velocity, hence it's not able to account for increased upscattering probability at energies slightly below a resonance. The second model was Doppler Broadening Rejection Correction (DBRC), an exact scattering model free of assumption mentioned previously. The comparison between "only SVT" approach and approach where DBRC was used for U238 scatterings ("SVT \& DBRC") is presented in the Table 1. We see that with nominal fuel

Table 1: Differences in pcm between pointwise $\mathrm{T} 4$ reference calculations with "only SVT" and "DBRC \& SVT" scattering treatment

\begin{tabular}{lllll}
\hline & $($ Only SVT $)-($ SVT \& DBRC) & ass. C1-2D & ass. C2-2D & core-3D \\
\hline $\mathbf{1 0 . 5 \%}$ Be & $\Delta \rho\left(900^{\circ} \mathrm{C}\right)$ & -5 & -2 & -8 \\
& $\Delta \rho\left(2000^{\circ} \mathrm{C}\right)$ & 45 & 37 & 49 \\
& $\Delta K_{\boldsymbol{D}}$ & 75 & 59 & 86 \\
\hline $\mathbf{3 . 5 \%} \mathbf{Z r H}_{\mathbf{2}}$ & $\Delta \rho\left(900^{\circ} \mathrm{C}\right)$ & 26 & 29 & 30 \\
& $\Delta \rho\left(2000^{\circ} \mathrm{C}\right)$ & 108 & 94 & 112 \\
& $\Delta K_{\boldsymbol{D}}$ & 125 & 99 & 124 \\
\hline \multirow{2}{*}{ no moderator } & $\Delta \rho\left(900^{\circ} \mathrm{C}\right)$ & -1 & -2 & 1 \\
& $\Delta \rho\left(2000^{\circ} \mathrm{C}\right)$ & 0 & -5 & 1 \\
& $\Delta K_{\boldsymbol{D}}$ & 1 & -5 & 1 \\
\hline
\end{tabular}

temperature applied the differences between the two models are insignificant for all three types of assembly. However when using a higher fuel temperature of $2000^{\circ} \mathrm{C}$ we observe pronounced differences reaching more than $100 \mathrm{pcm}$. Due to lack of resonance upscatterings in "only SVT" model the resonant absorptions in U238 are underestimated which results in higher reactivity. The highest differences were obtained in case of $\mathrm{ZrH}_{2}$ moderator due to the largest neutron population in the epithermal range, where upscattering occurs with the highest probability. For similar reasons we see greater differences for $\mathrm{C} 1$ assembly than $\mathrm{C} 2$, as higher $\mathrm{Pu}$ enrichment results in lower epithermal flux. Because of such a significant difference between $900^{\circ} \mathrm{C}$ and $2000^{\circ} \mathrm{C}$ the Doppler constant calculated between the two temperatures varies strongly between scattering models. The underestimation of Doppler constant reached up to $125 \mathrm{pcm}$ for $\mathrm{ZrH}_{2}$ moderator, more than 5\% of the value of $\mathrm{K}_{\mathrm{D}}$. Because of the strong differences obtained, all the calculations in the further sections included the improved upscattering treatment. In case of A3 we used a preprocessed nuclear data library with modified transfer matrices to account for the resonance upscattering.

\section{QUANTIFICATION OF BIASES WITH DETERMINISTIC-MONTE CARLO SCHEME}

An in-depth study and validation of lattice calculation of CADOR was already conducted in the past [8]. In order to expand the analysis, the purpose of this study is to perform the first validation of a full two-step calculation scheme, with particular focus on homogenization-condensation and core calculation. During homogenization-condensation process a part of the information about exact shape of the assemblies and neutron spectrum is lost and thus it can't be transferred to the core calculation. Therefor it is crucial to ensure that the simplified core model we use is still a good representation of the core that we try to study. In case of CADOR the treatment used for standard SFR i.e. homogenization of entire fuel assembly into one region and condensation into a 33 energy groups may lead to large numerical bias due to the following reasons: 
1) fully homogeneous assembly is less representative in case of lower neutron mean free path combined with heterogeneous moderator placement

2) lower mean neutron energy may require finer energy mesh in the lower part of the spectrum. In order to test these hypotheses our first approach was to use a hybrid calculation scheme that combines both deterministic and Monte Carlo methods. In the scheme the homogenized-condensed cross-sections are computed via series of A3 lattice calculations as in usual deterministic approach. The cross-sections are then transferred to T4 Monte Carlo code where the multigroup calculation with homogenized core geometry is performed, similarly to A3 core calculation. Such combined A3-T4 approach provides two major advantages: 1) the calculation is free of biases coming from deterministic core calculation solver and 2) it allows to test configurations that may not be feasible for deterministic solver due to computing limitations. By using this method we were able to conduct core calculations with three different homogenized models of fissile zones (Figure 3) and with both 33 energy groups as well as 1760 groups i.e. without condensation of cross-section. The remaining zones in the core were homogenized with HOM approach in all studied cases.

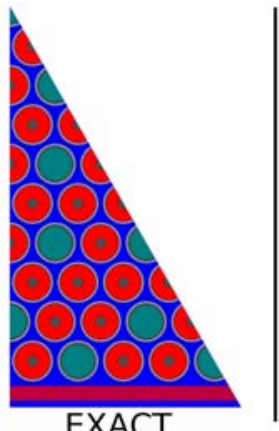

EXACT

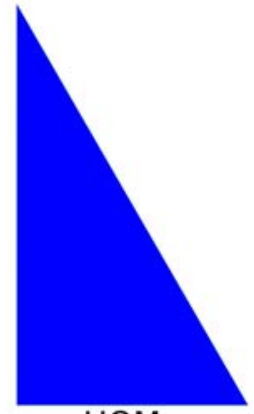

HOM

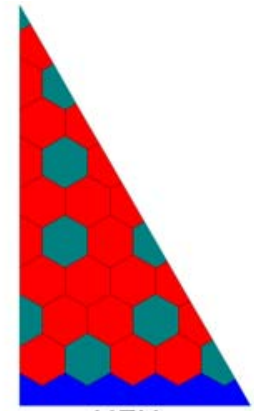

HEX

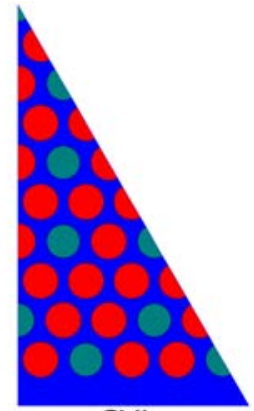

CYL

Figure 3: 2D view of exact geometry of $1 / 12^{\text {th }}$ of $11 \%$ Be CADOR fuel assembly (fissile zone) and its three homogenization patterns

\subsection{Global bias on core reactivity}

The global biases after entire two-step A3-T4 calculation with respect to T4 reference calculation are presented in Figures $4 \mathrm{a}$ and $4 \mathrm{~b}$. Both the condensation and homogenization have a visible

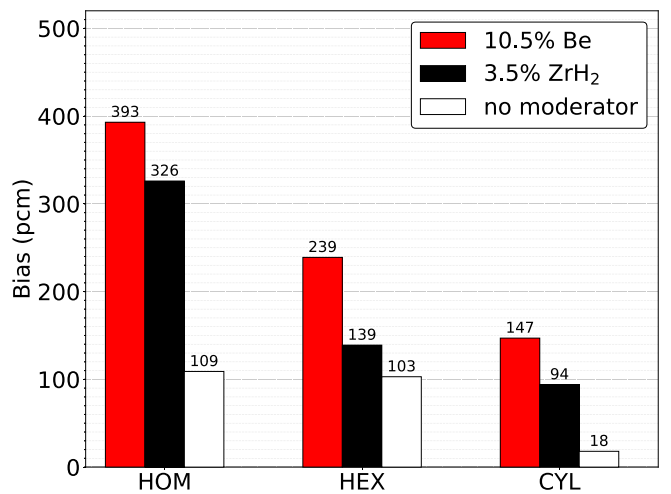

(a) 1760 energy groups (no condensation)

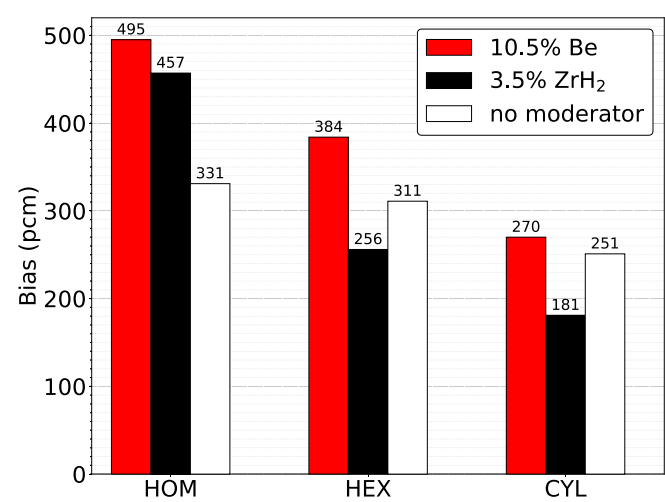

(b) 33 energy groups

Figure 4: Global core reactivity bias of A3-T4 scheme compared to T4 reference 
impact on the magnitude of the bias. For moderated cores the difference between homogenization patterns is much higher than in case without moderator. Regardless of the moderator used we can see a visible trend of bias reduction due to homogenization type with HOM being the worst case and the CYL being the best one. Using CYL pattern in place of HOM led to an improvement of 277 pcm for $\mathrm{ZrH}_{2}$-moderated core and $225 \mathrm{pcm}$ for Be-moderated core for 33 group mesh. In case of standard SFR a far lower bias reduction proves that CYL and HEX approaches are justified only in presence of moderator. The impact of condensation from 1760 groups to 33 is more pronounced in case of standard SFR - the global bias increases by about $200 \mathrm{pcm}$. On the other hand, in presence of moderator the energy condensation increases the bias by only around $90-140 \mathrm{pcm}$. As a result, for 33 energy groups the biases for all three cores are comparable if HEX or CYL patterns are used.

\subsection{Bias on Doppler constant and void coefficient}

If we look at the bias of Doppler feedback and void coefficient (Table 2) we can notice that the values are very weakly dependent on the type of homogenization. The magnitude of Doppler Effect was represented as a Doppler constant $\mathrm{K}_{\mathrm{D}}$, calculated for fuel temperatures of $900^{\circ} \mathrm{C}$ and $2000^{\circ} \mathrm{C}$. Since both reactivity feedbacks requires calculation of core in two different states the biases of each individual calculation are compensated. As a result the discrepancies from reference are very low and do not exceed $70 \mathrm{pcm}$ in case of $\mathrm{K}_{\mathrm{D}}$ and $100 \mathrm{pcm}$ in case of void coefficient.

Table 2: Bias on Doppler constant and void coeff. in pcm for A3-T4 scheme (33 groups in T4) compared to $\mathrm{T} 4$ reference

\begin{tabular}{|c|c|c|c|c|c|c|}
\hline & \multicolumn{2}{|c|}{ HOM } & \multicolumn{2}{|c|}{ HEX } & \multicolumn{2}{|c|}{ CYL } \\
\hline & $\mathbf{K}_{\mathrm{D}}$ & Void & $\mathbf{K}_{\mathrm{D}}$ & Void & $\mathbf{K}_{\mathrm{D}}$ & Void \\
\hline $10.5 \% \mathrm{Be}$ & -45 & 67 & -67 & 73 & -59 & 30 \\
\hline $3.5 \% \mathrm{ZrH}_{2}$ & 9 & 78 & -31 & 92 & -49 & 34 \\
\hline no moderator & -45 & 94 & -27 & 96 & -49 & 50 \\
\hline
\end{tabular}

T4 reference absolute values

\begin{tabular}{c|c}
$\mathrm{K}_{\mathrm{D}}$ & Void \\
\hline$-2438 \mathrm{pcm}$ & $929 \mathrm{pcm}$ \\
$-2365 \mathrm{pcm}$ & $1070 \mathrm{pcm}$ \\
$-856 \mathrm{pcm}$ & $1737 \mathrm{pcm}$
\end{tabular}

\subsection{Bias on control rods worth}

For this study we defined control rods worth as a reactivity difference between core with control rods fully extracted and inserted (safety rods are excluded) up to the bottom of the fissile zone. The quantification of global bias on the rod's worth is presented in Table 3. A much bigger bias

Table 3: Biases on control rods worth in pcm for A3-T4 scheme (33 groups in T4) compared to $\mathrm{T} 4$ reference

\begin{tabular}{lllllllll}
\hline & \multicolumn{2}{c}{ HOM } & & \multicolumn{2}{c}{ HEX } & & \multicolumn{2}{c}{ CYL } \\
\cline { 2 - 3 } \cline { 8 - 9 } \cline { 8 - 9 } & P1 & P3 & & P1 & P3 & & P1 & P3 \\
\hline $\mathbf{1 0 . 5 \%}$ Be & 894 & 492 & & 621 & 520 & & 240 & 262 \\
$\mathbf{3 . 5 \%} \mathbf{Z r H}$ & 606 & 284 & & 393 & 314 & & 130 & 147 \\
no moderator & 226 & 154 & & 202 & 190 & & 15 & 72 \\
\hline
\end{tabular}

T4 reference absolute value

Control rods worth

$-8328 \mathrm{pcm}$

$-5838 \mathrm{pcm}$

$-6830 \mathrm{pcm}$

on cores reactivity after the insertion leads to a high bias on control rods worth. The difference 
between CADOR cores and standard SFR core is again very pronounced here. We observe two potential ways of bias reduction. Using CYL and HEX fuel assembly models decreases the bias significantly, despite the control rods remaining completely homogeneous. An improvement can be also achieved by increasing scattering anisotropy by using Legendre polynomials of order up to P3. A visible improvement is achieved for moderated cores especially in case of Be moderator.

\section{APOLLO3 ${ }^{\circledR}$ CORE CALCULATION}

After the study of different biases with A3-T4 method the purpose of this section is to present a number of results obtained with regular two-step A3 scheme with core calculation handled by MINARET solver. The bias quantification calculated with A3 code are visible in Figure 5. The
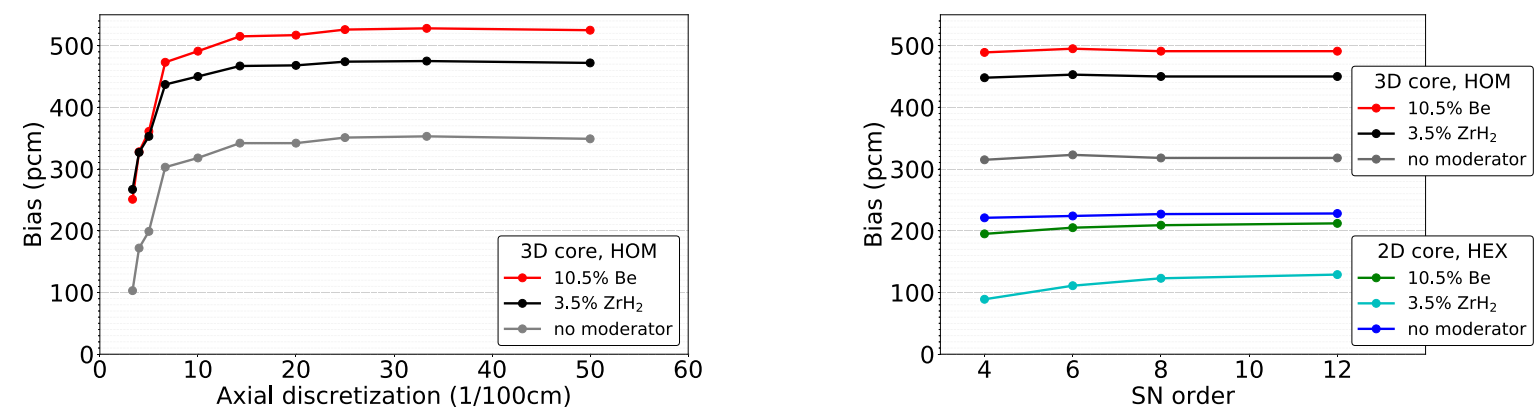

Figure 5: Bias of A3 calculation compared to T4 reference calculation

results are presented for a much more limited set of calculations compared to studies with A3-T4 scheme due to enormous computational costs. The computational complexity increases rapidly with number of finite elements. More heterogeneous geometries imply more refined spatial meshing, thus CYL pattern is not a feasible choice for geometry as large as the core we investigate. In case of HEX pattern the calculations were successfully performed with a simplified 2D core model (axially homogeneous, without blankets and gas plenums). The results are presented as a function of some of the crucial solver parameters. We can see that with sufficiently refined solver parameters the A3 results converged to those of A3-T4 from section 5, as the second step in both A3 and A3-T4 scheme used the same set of homogenized-condensed cross-sections. The values convergence in function of axial discr. exhibit a similar behavior regardless of the moderation type. In case of SN order no accuracy improvement is seen for calculations with HOM homogenization, however a slight improvement can be seen for HEX, particularly when $\mathrm{ZrH}_{2}$ moderator is used. We can conclude (based also on results out of scope of this article) that CADOR doesn't require different MINARET settings than a standard SFR, with exception of anisotropy order and SN order in some cases.

\section{CONCLUSIONS}

This paper aimed to quantify the global numerical biases of a deterministic two-step calculation scheme in case of moderated SFR core (CADOR), with focus on investigation of homogenizationcondensation procedure and full core calculation. We proved that because of the high importance of resonance upscattering a accurate treatment of neutron scattering is strongly advised for fuel temperatures higher than nominal. The approach we used, combining lattice calculation in A3 with 
multigroup core calculation in T4, allowed us to study in details complex semi-heterogeneous geometries even with very fine energetic mesh. The results suggest that standard calculation scheme for fast reactors leads to stronger numerical bias for CADOR core than a standard SFR core, especially in case of control rod worth. However, by applying a number of improvements (more heterogeneous assembly description, higher Legendre polynomials expansion) the bias can be reduced to a level of SFR core. Some of the results were reproduced with purely deterministic A3 calculation scheme, achieving very good agreement with A3-T4 scheme. However due to the limitations of computing power some of the studied homogenization patterns proved to not be feasible for deterministic calculations with core geometry of this scale. The study shown that global bias of CADOR does not deviate significantly from other SFR designs, and therefore a validation with more advanced bias breakdown is not required. In the near future a A3 core calculation with 3D core geometry and HEX homogenization type will be studied. It is also of interest to study the more computationally demanding schemes with smaller geometry of SMR type CADOR core.

\section{ACKNOWLEDGEMENTS}

The authors wish to thank EDF and FRAMATOME for their financial support of this work.

\section{REFERENCES}

[1] A. Zaetta et al., "CADOR Core with Adding DOppleR effect concept application to sodium fast reactors," EPJ Nuclear Sci. Technol., 5, pp. 1-13 (2019)

[2] H. Golfier et al., APOLLO3: a common project of CEA, AREVA and EDF for the development of a new deterministic multi-purpose code for core physics analysis, Proceedings of International Conference on Mathematics, Computational Methods and Reactor Physics (M\&C 2009), Saratoga Springs, New York, USA, May 3-7 (2009).

[3] P. Archier et al. Validation of the Newly Implemented 3D TDT-MOC Solver of APOLLO3 Code on a Whole 3D SFR Heterogeneous Assembly, Proceedings of PHYSOR 2016: Unifying Theory and Experiments in the 21st Century, Sun Valley, Idaho, USA, May 1-5 (2016)

[4] V. Jouault et al., "A New Breakdown Methodology to Estimate Neutronic Model Biases Applied to APOLLO3 SFR Core Calculations," Proceedings of International Conference on Mathematics and Computational Methods Applied to Nuclear Science and Engineering (M\&C 2017), Jeju, Korea, 16-20 April 2017

[5] J.-Y. Moller and J.-J. Lautard. MINARET, a Deterministic Neutron Transport Solver for Nuclear Core Calculations, International Conference on Mathematics and Computational Methods Applied to Nuclear Science and Engineering (M\&C 2011), Rio de Janeiro, Brazil, May 8-12 (2011)

[6] E. Brun et al. TRIPOLI-4, CEA, EDF and AREVA reference Monte Carlo code, Annals of Nuclear Energy, 82, pp. 151160 (2015)

[7] M. Ouisloumen, R. Sanchez, "A Model for Neutron Scattering Off Heavy Isotopes That Accounts for Thermal Agitation Effects," Nuclear Science and Engineering, 107, pp. 189200 (1991)

[8] M. Zajaczkowski et al., "Analysis of calculation scheme of APOLLO3 ${ }^{\circledR}$ applied to moderated SFR fuel assembly," Proceedings of International Congress on Advances in Nuclear Power Plants, Juin les Pins, France (2019) 